Aim of the study: Recent advances in chemotherapy have increasingly enabled conversion surgery (CS) in patients with initially unresectable pancreatic cancer $(P C)$, but patient selection remains controversial. We examined the characteristics of patients who would benefit from this procedure.

Material and methods: The clinical and pathological data of 38 patients with unresectable PC, who underwent CS after a favourable response to chemo(radio)therapy at our institute, were investigated. Univariate and multivariate analyses were performed to identify predictors for overall survival (OS). Several inflammation-based scores, such as the modified Glasgow prognostic score (mGPS), were also evaluated.

Results: The patients included 13 with locally advanced disease and 25 with metastatic disease. After non-surgical treatment with a median duration of six months, 27 patients $(71 \%)$ underwent $\mathrm{RO} / 1$ resection, and the remainder underwent $\mathrm{R} 2$ resection. The twoyear and five-year OS from the initial treatment for all patients were $64 \%$ and $29 \%$, respectively, and the median survival was 29.1 months. Univariate analysis showed that age $<62$ years, preoperative CA19-9 decrease rate $\geq 89 \%$, preoperative mGPS-0, and RO/ 1 resection were related to a favourable OS. RO/1 resection and mGPS-0 were independent prognostic factors according to multivariate analysis.

Conclusions: Preoperative mGPS is a potential predictor of survival and can aid selection of patients for whom CS could yield promising prognosis for initially unresectable PC.

Key words: pancreatic cancer, conversion surgery, modified Glasgow prognostic score.

Contemp Oncol (Pozn) 2018; 22 (4): 229-235 DOI: https://doi.org/10.5114/wo.2018.81344

\section{The preoperative modified Glasgow prognostic score for the prediction of survival after pancreatic cancer resection following non-surgical treatment of an initially unresectable disease}

\author{
Shinichi lkuta' ${ }^{1}$ Takashi Sonoda ${ }^{2}$, Tsukasa Aihara' ${ }^{1}$ Takayoshi Nakajima ${ }^{1}$, \\ Naoki Yamanaka ${ }^{1}$
}

${ }^{1}$ Department of Surgery, Meiwa Hospital, Japan

${ }^{2}$ Department of Medical Oncology, Meiwa Hospital, Japan

\section{Introduction}

Pancreatic cancer (PC) is a lethal malignant tumour and has a poor prognosis. According to the latest cancer statistics published in 2018, PC is the seventh leading cause of cancer death in both males and females worldwide, with almost as many deaths $(n=432,000)$ as cases $(n=459,000)$ [1]. A projection study estimated that PC would escalate from the fourth to the second leading cause of cancer deaths in the United States in 2030 [2]. Surgical resection remains the preferred treatment option for localised, resectable PC because it offers the only chance for a cure. However, because of the lack of efficient and reliable screening methods for the early detection of PC and the lack of clinical signs and symptoms, except for diabetes development, especially in advanced ages, more than $80 \%$ of patients present with advanced locoregional or metastatic disease, which results in low rates of resection and survival [3].

The cornerstone of treatment for locally advanced or metastatic PC is palliative chemotherapy. Fortunately, recent advances in systemic chemotherapy have led to the selection of patients with unresectable disease where surgery may be potentially indicated. During the last decade, reports of an additional surgery, called conversion surgery (CS), after favourable response to chemotherapy or chemoradiotherapy for initially unresectable PC have been increasing [4-10]. In several studies, the prognosis of patients who underwent CS was significantly better than that of patients who received non-surgical treatment alone $[6,7,9]$.

Gemcitabine plus nab-paclitaxel and FOLFIRINOX (oxaliplatin, irinotecan, fluorouracil plus leucovorin) are the first-line chemotherapy for unresectable PC [11, 12]. Since 2008, in an appropriate clinical setting, we have conducted modified chemotherapy using gemcitabine in combination with taxane and platinum agents for patients with advanced or recurrent PC. Patients were administered itraconazole (ITCZ), a commonly used antifungal agent that potently inhibits the Hedgehog pathway, P-glycoprotein, and angiogenesis [13-15]. We previously reported promising results with this intense combination therapy to treat PC patients with metastatic disease [16]. Some initially unresectable PC patients, even those with multiple metastatic lesions, have received tumour resection after down-staging or disease control with chemotherapy at our institute.

While no data regarding the role of CS from randomised trials are available, surgery-combined multidisciplinary therapy may improve outcomes of 
advanced PC patients. However, determining who might benefit from CS remains controversial because some patients relapse early after CS and consequently have a dismal prognosis. In the present study, we aimed to explore preoperative predictors of survival in patients with unresectable PC, who underwent CS following non-surgical treatment in order to identify the determinant in selecting patients for CS.

\section{Material and methods}

\section{Patients and definition of unresectable pancreatic cancer}

We enrolled 43 patients with unresectable PC, who had responded well to chemo(radio)therapy and consequently underwent surgical exploration at our institute between February 2011 and June 2018. Five patients who underwent pancreatectomy intending palliative resection were excluded, and finally 38 patients were analysed. At a baseline evaluation, tumour status was assessed using various imaging techniques, including multidetector row computed tomography, ultrasonography, magnetic resonance imaging, and positron emission tomography (PET). Unresectable PC was defined and subclassified as locally advanced or metastatic according to the general rules for

Table 1. Patient baseline characteristics and preoperative non-surgical treatment

\begin{tabular}{|c|c|}
\hline Characteristics & Results \\
\hline Age, years & $64(39-79)$ \\
\hline Sex (male/female), $n$ & $20 / 18$ \\
\hline Main tumour location (head/body-tail), $n$ & $21 / 17$ \\
\hline $\begin{array}{l}\text { Reason for unresectability, } n \\
\text { Locally advanced* } \\
\text { SMA } \\
\text { CA-CHA } \\
\text { CHA-PHA } \\
\text { SMV-PV } \\
\text { Metastatic* } \\
\text { Liver } \\
\text { Para-aortic lymph nodes } \\
\text { Peritoneum }\end{array}$ & $\begin{array}{c}13 \\
6 \\
4 \\
3 \\
2 \\
25 \\
17 \\
5 \\
4\end{array}$ \\
\hline $\begin{array}{l}\text { Preoperative non-surgical treatment } \\
\text { Chemotherapy, } n \\
\text { First-line } \\
\text { Combination chemotherapy with ITCZ } \\
\text { Gem + nab-paclitaxel } \\
\text { Gem + Erlotinib } \\
\text { Gem + S1 } \\
\text { Gem } \\
\text { FOLFIRINOX } \\
\text { Second-line } \\
\text { Combination chemotherapy with ITCZ } \\
\text { Radiotherapy, } n \\
\text { RECIST (CR/PR/SD), } n \\
\text { Treatment duration, months }\end{array}$ & $\begin{array}{c}6 \\
5 \\
0 / 26 / 12 \\
6(3-48)\end{array}$ \\
\hline $\begin{array}{l}\text { *There were overlapping reasons for unresectability. } \\
\text { SMA - superior mesenteric artery; CA - celiac artery; } \\
\text { artery; PHA - proper hepatic artery; SMV - superior } \\
\text { bortal vein; ITCZ - itraconazole; Gem - gemcitabine; } \\
\text { irinotecan, fluorouracil plus leucovorin; RECIST - Resh } \\
\text { Solid Tumours; CR - complete response; PR - partial }\end{array}$ & $\begin{array}{l}\text { non hepatic } \\
\text { ein; PV- } \\
\text { - oxaliplatin, } \\
\text { ation Criteria in } \\
\text { - stable disease }\end{array}$ \\
\hline
\end{tabular}

the study of pancreatic cancer by the Japan Pancreas Society (seventh edition) [17] as follows: locally advanced was defined as: 1) superior mesenteric vein/portal vein: tumour contact/invasion of 180 or more degrees/occlusion, exceeding the inferior border of the duodenum; 2) superior mesenteric artery, celiac artery: tumour contact/invasion of 180 or more degrees; 3) common hepatic artery: tumour contact/invasion showing tumour contact/invasion of the proper hepatic artery and/or celiac artery; or 4) aorta: tumour contact or invasion. Metastatic was defined as a distant metastasis including peritoneal spread, involvement of para-aortic or extra abdominal lymph nodes, lung, bone, or liver metastasis [17].

\section{Combination chemotherapy with itraconazole}

First-line chemotherapy included modified combination therapy with ITCZ in 29 (76.3\%) of the patients (Table 1). Six of the remaining nine patients received the treatment as a second-line setting. Briefly, on day 1 of the treatment, the starting doses of intravenous gemcitabine, docetaxel, and carboplatin were $1000 \mathrm{mg} / \mathrm{m}^{2}, 35 \mathrm{mg} / \mathrm{m}^{2}$, and $4 \mathrm{mg} \cdot \mathrm{min}^{-1} \cdot \mathrm{ml}^{-1}$ (area under the curve), respectively. An oral ITCZ solution was also administered at a daily dose of $400 \mathrm{mg}$ (days -2 to 2). This regimen was repeated every two weeks. Since December 2016, docetaxel and carboplatin were replaced by nab-paclitaxel $\left(125 \mathrm{mg} / \mathrm{m}^{2}\right)$ and oxaliplatin $\left(85 \mathrm{mg} / \mathrm{m}^{2}\right)$, respectively, based on the approval of gemcitabine plus nab-paclitaxel and FOLFIRINOX for advanced PC (registered in UMIN-CTR [UMIN 000025398]). Chemotherapy dose reduction and/or dose delay were used as appropriate in response to toxicity. Granulocyte colony stimulating factor was administered according to the general recommendations until absolute neutrophil counts recovered. The tumour response was objectively assessed according to the Response Evaluation Criteria in Solid Tumours (RECIST) version 1.1 [18].

\section{Indication of conversion surgery}

Surgical exploration was considered according to the following characteristics: 1) administration of chemotherapy for three months or more; 2) primary tumour response of stable disease or better; 3) significant shrinkage or disappearance of metastases without appearance of new lesions; 4) reduction of serum carbohydrate antigen 19-9 (CA19-9), which is the most commonly used biomarker for monitoring therapeutic progress in PC; 5 ) decrease in the standardised uptake value of [18F] fluorodeoxyglucose by PET; 6) an Eastern Cooperative Oncology Group performance status $\leq 1$; and 7 ) adequate organ function. Specifically, patients with remaining metastatic liver disease after non-surgical treatment were considered for CS if all visible lesions were deemed potentially operable regardless of the size, number, or location of the liver tumours (e.g. wedge resection for superficial lesions and/or thermal ablation for deeper lesions).

\section{Data collection}

Clinical and pathological data were obtained from patients' notes and from the oncology database. Using blood 
data obtained before treatment initiation and within a few days before surgery, several inflammation-based prognostic scores were evaluated to determine predictors of overall survival (OS). No clinical evidence of infection or other inflammatory conditions were documented at the time of blood sampling. The modified Glasgow prognostic score (mGPS) was calculated as follows: patients with a normal albumin level $(\geq 3.5 \mathrm{~g} / \mathrm{dl})$ and C-reactive protein (CRP) $(\leq 0.5 \mathrm{mg} / \mathrm{dl})$ were scored as 0 , those with both low albu$\min (<3.5 \mathrm{~g} / \mathrm{dl})$ and high CRP $(>0.5 \mathrm{mg} / \mathrm{dl})$ were scored as 2 , and those with only low albumin $(<3.5 \mathrm{~g} / \mathrm{dl})$ or high CRP (> $0.5 \mathrm{mg} / \mathrm{dl}$ ) were scored as 1 [19]. The neutrophil-to-lymphocyte ratio and the platelet-to-lymphocyte ratio were defined as the ratio of the absolute peripheral blood neutrophil and lymphocyte counts and the absolute platelet and lymphocyte counts, respectively. The prognostic nutritional index was calculated as $(10 \times$ albumin $)+(0.005 \times$ lymphocyte count). The Clavien-Dindo (C-D) grade classification was used for defining morbidity and mortality [20]. The pathological effect of preoperative treatment was assessed by the Evans grading system [21]. Resection curability was classified as follows: RO, no residual tumour; R1, microscopic residual tumour; R2, macroscopic residual tumour.

\section{Ethics statement}

Details of the therapeutic options were given to the patients, and written informed consent was obtained before any treatment was undertaken. This study was approved by the institutional Ethics Committee for a retrospective analysis of the collected data in accordance with the ethical standards of the World Medical Association's Declaration of Helsinki.

\section{Statistical analysis}

Quantitative variables were expressed as a median value and range. OS was calculated from the date of treatment initiation to that of death or lost contact with the patient. The median follow-up time was calculated using the reverse Kaplan-Meier method [22]. The time-dependent receiver operating characteristic curve was generated to estimate the optimal cut-off values of quantitative variables for the median os prediction. The variables were considered valid candidates for survival analysis if the area under the curve was > 0.6 [23]; the cut-off value was selected according to the maximum sum of sensitivity and specificity. Survival curves were estimated by the Kaplan-Meier method and compared, for univariate analysis, by the log-rank test. Multivariate analysis was performed using the Cox multivariate proportional hazard regression model in a stepwise manner. All statistical analyses were performed with R Statistical Software (Foundation for Statistical Computing, Vienna, Austria), and $p<0.05$ was considered significant.

\section{Results}

\section{Patient characteristics and non-surgical} treatments

The baseline characteristics of 38 patients are summarised in Table 1. There were 20 male and 18 female
Table 2. Pre-treatment, preoperative variables, and AUCs for survival prediction

\begin{tabular}{lccc}
\hline Parameter & AUC & $\begin{array}{c}\text { Cut-off } \\
\text { value }\end{array}$ \\
Pre-treatment & & & \\
mGPS $(0 / 1 / 2), n$ & $30 / 7 / 1$ & & \\
NLR & $227(102-700)$ & 0.388 & NA \\
PLR & $136(58-655)$ & 0.474 & NA \\
PNI & $47(30-57)$ & 0.544 & NA \\
CA19-9 (U/ml) & $615(58-75746)$ & 0.446 & NA \\
Preoperative & & & \\
mGPS $(0 / 1 / 2), n$ & $31 / 2 / 5$ & & \\
NLR & $193(75-700)$ & 0.309 & NA \\
PLR & $143(70-437)$ & 0.364 & NA \\
PNI & $44(31-57)$ & 0.443 & NA \\
CA19-9 (U/ml) & $74(7.7-4482)$ & 0.565 & NA \\
CA19-9 decrease rate (\%) & $89.4(10.1-99.9)$ & 0.632 & 89
\end{tabular}

AUC - area under the curve; $m G P S$ - modified Glasgow prognostic score; $N L R$ - neutrophil-to-lymphocyte ratio; $P L R$ - platelet-to-lymphocyte ratio; $P N I$ - prognostic nutritional index; CA19-9 - carbohydrate antigen 19-9; NA - not adopted

patients with a median age of 64 years (range 39-79 years). The reason for unresectability was 13 locally advanced tumours and 25 distant organ metastases. The most frequent metastatic site was liver $(n=17)$ followed by para-aortic lymph node $(n=5)$. The first-line chemotherapy other than the ITCZ-containing regimen included combinations of gemcitabine plus nab-paclitaxel $(n=5)$, gemcitabine plus erlotinib $(n=1)$, gemcitabine plus S1 $(n=1)$, gemcitabine alone $(n=1)$, or FOLFIRINOX $(n=1)$. Additional radiotherapy to improve local tumour control was administered in five patients with locally advanced PC infiltrating surrounding artery since December 2016. After preoperative treatment with a median duration of six months, serum CA19-9 level decreased from 615 to $74 \mathrm{U} / \mathrm{ml}$, with $61 \%$ of patients having $>80 \%$ reduction (Table 2 ). A radiological response of complete response, partial response, and stable disease was found in 0, 26, and 12 patients, respectively.

\section{Surgical outcomes}

A potentially curative surgery was attempted for all patients. The operative procedures included conventional pancreatoduodenectomy (PD) in one patient, subtotal stomach-preserving PD in 20 patients, and distal pancreatectomy in 17 patients. Concomitant major vascular resection was performed in 12 patients, and the details are listed in Table 3. Three patients required combined arterial and portal resection. Thirteen patients underwent metastatic liver resection, and the most common procedure was a wedge resection. Of these, five patients underwent liver resection alone, and eight patients underwent combined resection and intra-operative ablation for multiple metastatic lesions. Two patients underwent intra-operative ablation alone. Twenty-one (55.3\%) patients had an RO resection, six underwent $\mathrm{R} 1$ resection, and the remaining 11 had an R2 resection. There was one in-hospital mortality, where the patient died 62 days after surgery (distal pancreatectomy with celiac-axis resection) because of rapid peritoneal tumour dissemination. Including this 
Table 3. Surgical and pathological outcomes

\begin{tabular}{|c|c|c|}
\hline Operation type, $n$ & $\begin{array}{l}\text { PD } \\
\text { SSPPD } \\
\text { DP } \\
\text { DP-CAR }\end{array}$ & $\begin{array}{c}1 \\
20 \\
14 \\
3\end{array}$ \\
\hline Combined resections ${ }^{*}, n$ & $\begin{array}{c}\text { SMV-PV } \\
\text { CA-CHA } \\
\text { CHA-PHA } \\
\text { Adrenal gland } \\
\text { Liver (ablation) } \\
\text { Colon } \\
\text { Stomach }\end{array}$ & $\begin{array}{c}10 \\
3 \\
2 \\
2 \\
13(10) \\
2 \\
2\end{array}$ \\
\hline Operative time (min) & & $539(269-702)$ \\
\hline Operative blood loss (ml) & & $895(60-1860)$ \\
\hline Intraoperative transfusion, $n$ & Yes/No & $12 / 26$ \\
\hline Morbidity, $n(\%)$ & $\begin{array}{l}\text { Clavien-Dindo } \\
\text { grade } \geq \text { IIIa }\end{array}$ & $15(39.5)$ \\
\hline Mortality, $n(\%)$ & & $1(2.6)$ \\
\hline $\begin{array}{l}\text { Pathological findings, } n \\
\text { Histologic differentiation } \\
\text { pT } \\
\text { pN } \\
\text { pTNM stage }\end{array}$ & $\begin{array}{l}\text { Well/Moderate/ } \\
\text { Poor/UD } \\
\text { 1/2/3/4 } \\
0 / 1 \\
\text { I/II/III/IV }\end{array}$ & $\begin{array}{c}6 / 29 / 2 / 1 \\
11 / 5 / 15 / 7 \\
17 / 21 \\
2 / 10 / 4 / 22\end{array}$ \\
\hline $\begin{array}{l}\text { Evans grade } \\
\text { Primary tumour } \\
\text { Metastatic tumour }\end{array}$ & $\begin{array}{l}\text { I/IIa/IIb/III/IV } \\
1 /\|\mathrm{I} /\| \mathrm{lb} / \mathrm{II} / \mathrm{IV}\end{array}$ & $\begin{array}{l}4 / 3 / 17 / 13 / 1 \\
2 / 0 / 3 / 8 / 4\end{array}$ \\
\hline Residual tumour, $n$ & $\mathrm{RO} / 1 / 2$ & $21 / 6 / 11$ \\
\hline Adjuvant chemotherapy, $n$ (\%) & & $33(86.8)$ \\
\hline Recurrence, $n(\%)$ & & $25(65.8)$ \\
\hline \multicolumn{3}{|c|}{$\begin{array}{l}\text { *There were overlapping resections. } \\
P D \text { - pancreatoduodenectomy; SSPPD - subtotal stomach-preserving } \\
\text { pancreatoduodenectomy; DP - distal pancreatectomy; DP-CAR - distal } \\
\text { pancreatectomy with celiac-axis resection; SMV - superior mesenteric vein; } \\
P V \text { - portal vein; } C A \text { - celiac artery; CHA - common hepatic artery; } \\
P H A \text { - proper hepatic artery; } U D \text { - undefined }\end{array}$} \\
\hline
\end{tabular}

patient, a morbidity of C-D grade $\geq$ IIIa was observed in 15 patients (39.5\%). Pancreatic ductal adenocarcinoma was observed histologically in all patients, except one with locally advanced PC, which showed complete pathological response. A pathological response of Evans grade $\geq$ III for primary pancreatic tumour was noted in 14 patients (36.8\%), and that for metastatic tumour was noted in $12(70.6 \%)$ of 17 patients who underwent metastatic resection.

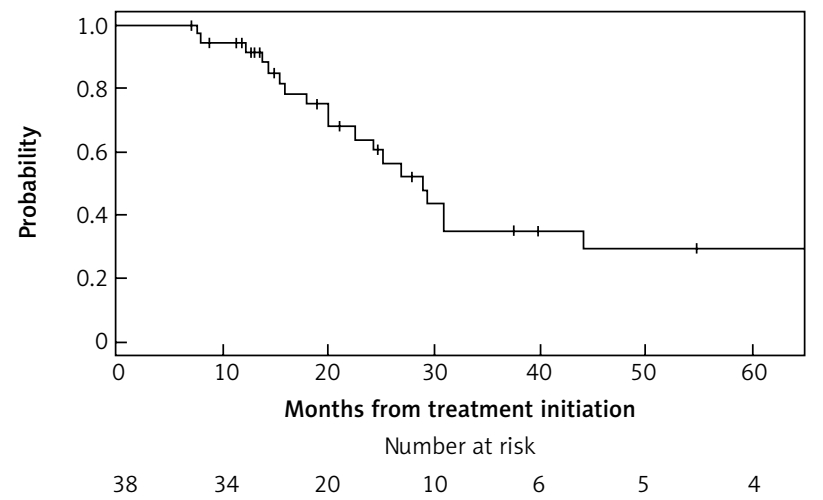

Fig. 1. Kaplan-Meier plots showing the overall survival of the patients

\section{Survival outcomes and significant predictors of overall survival}

During the median follow-up of 39.8 months (95\% confidence interval [Cl]: 24.6 - unavailable months), 22 (57.9\%) patients died, and the median OS was 29.1 months (95\% Cl: 20-44.2 months). The two-year and five-year OS rates from treatment initiation were $64 \%$ and $29 \%$, respectively (Fig. 1). The median survival after CS was 16.2 months (95\% Cl: 12.7-28 months) with a two-year survival of $35.9 \%$. Four patients survived longer than five years from treatment initiation, and all of them had received combination chemotherapy with ITCZ as a first-line or second-line treatment. Kaplan-Meier analysis and log-rank test showed that the OS of patients with age $<62$ years, preoperative CA19-9 decrease rate $\geq 89 \%$, preoperative mGPS-0, and RO/1 resection were significantly higher than other patients (Fig. 2). There was no evidence that other factors, including the reason for unresectability (locally advanced vs. metastatic), preoperative treatment duration, RECIST response, inflammation-based scores other than preoperative mGPS, pathological stage, or Evans grade, significantly influenced prognosis (Tables 2, 4). In multiple regression analyses, preoperative mGPS-0 (hazard ratio (HR): 0.291, 95\% Cl: $0.092-$ $0.927, p=0.037$ ), and R0/1 resection (HR: $0.323,95 \% \mathrm{Cl}$ : $0.124-0.840, p=0.021)$ were independent predictors of favourable OS (Table 4).

\section{Discussion}

The conversion surgery is increasingly recognised as a new therapeutic strategy for initially unresectable PC. When chemotherapy has produced a transient response and curative surgery can be accomplished, CS is occasionally associated with prolonged survival in selected patients [4-10]. However, because the relevant publications are only from retrospective case series, the survival benefits of CS have not been sufficiently investigated, and no evidence-based recommendation or consensus is available. A multi-institutional prospective observational study to evaluate the safety and efficacy of CS is already ongoing in Japan (Prep-04 study; UMIN000017793).

With intensive chemotherapy, the survival of unresectable PC patients has markedly improved, even in those who did not receive CS. There are reports of clinical outcomes of around 15 months or longer survival time in unresectable $P C$ patients receiving gemcitabine plus erlotinib [24], gemcitabine plus nab-paclitaxel [25], or FOLFIRINOX [26] with or without combination of radiotherapy. In a population-based study from Ontario, 12 (8.2\%) out of 147 PC patients with locally advanced disease underwent CS after initial chemotherapy including FOLFIRINOX and gemcitabine plus nab-paclitaxel, but CS was not associated with better survival in a multivariable analysis [27]. Thus, CS remains an individual approach for selected patients only, and clarification of the characteristics of patients who would benefit from CS is an important clinical matter.

To the best of our knowledge, this is the first study to show an independent predictive ability of mGPS on the survival outcome in patients undergoing CS. The median OS of patients with mGPS-1 or mGPS-2 preoperatively was 

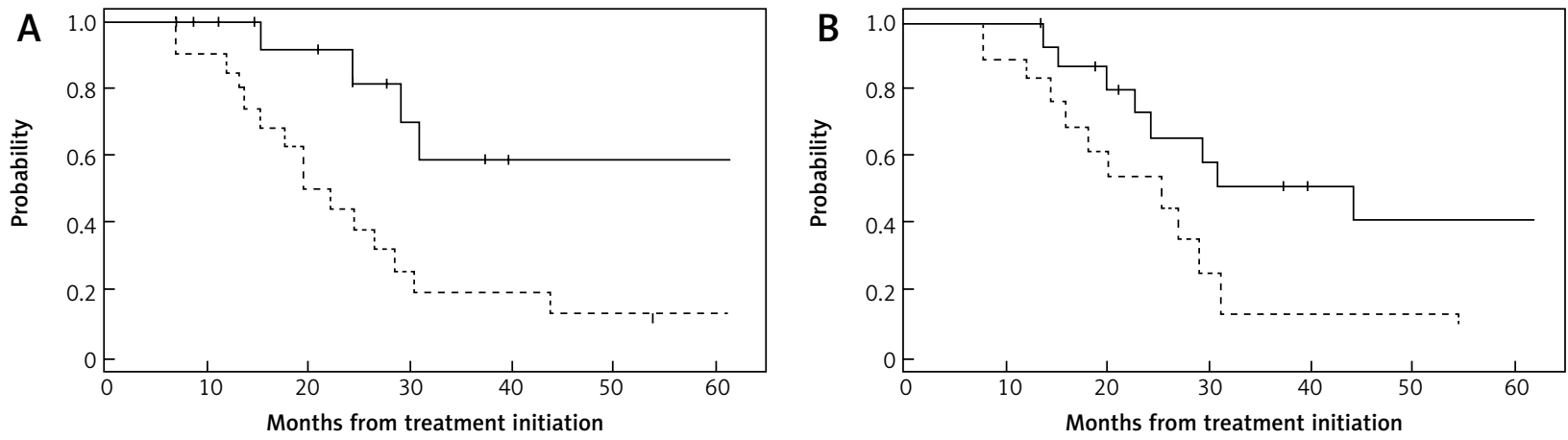

\begin{tabular}{cccccccc}
$<62$ & 15 & 14 & 11 & 6 & 3 & 3 & 3 \\
$\geq 62$ & 23 & 20 & 9 & 4 & 3 & 2 & 1 \\
& & \multicolumn{7}{c}{ Age (years) }
\end{tabular}

\begin{tabular}{ccccccc}
$\geq 89 \% 17$ & 17 & 12 & 8 & 5 & 4 & 4 \\
$<89 \% 21$ & 17 & 8 & 2 & 1 & 1 & 0 \\
& \multicolumn{7}{c}{ CA19-9 decrease rate (\%) } \\
& $-\geq 89$ & $-->89$
\end{tabular}
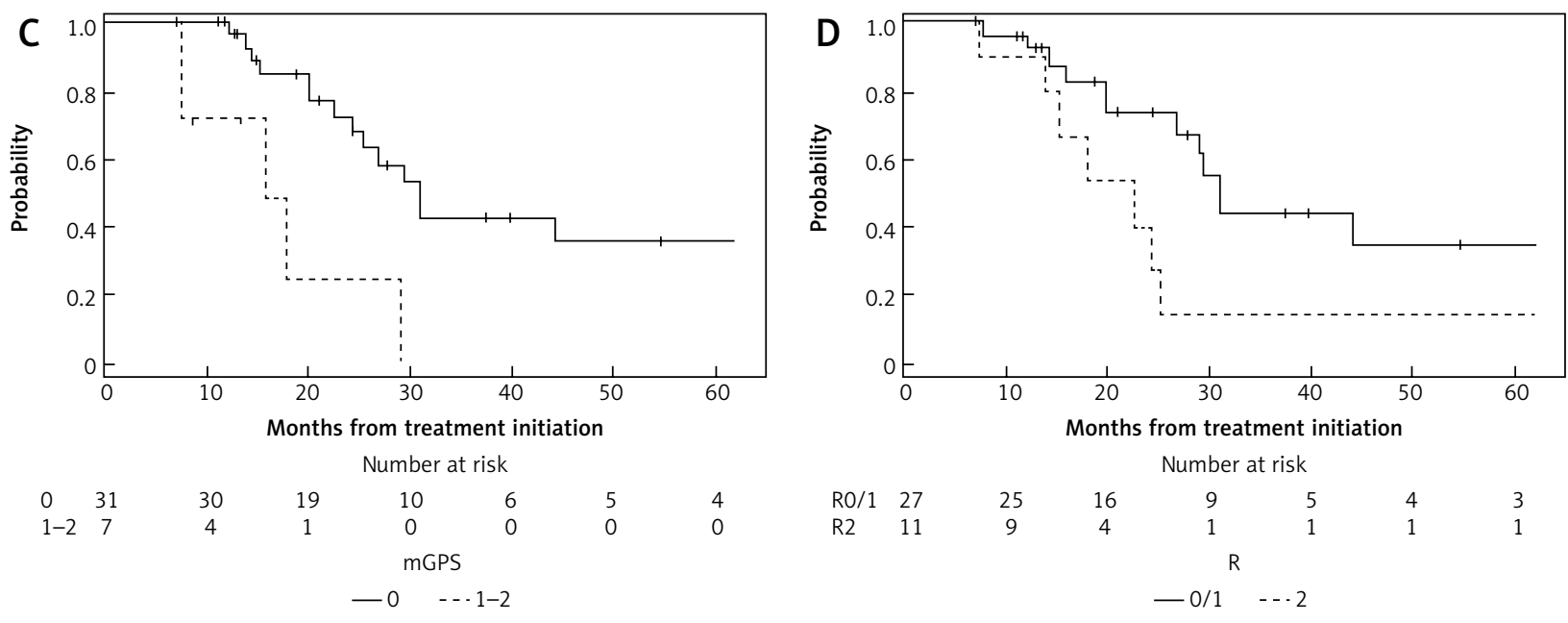

Fig. 2. Kaplan-Meier plots for overall survival by subgroups; (A) Age $\geq 62$ years vs. $<62$ years $(p=0.038)$; (B) Carbohydrate antigen $19-9$ decrease rate $\geq 89 \%$ vs. $<89 \%(p=0.047)$; (C) mGPS-0 vs. $1-2(p=0.002)$; (D) R0/1 resection vs. R2 resection $(p=0.033)$

Table 4. Univariate and multivariate analyses for overall survival

\begin{tabular}{|c|c|c|c|c|}
\hline \multirow[t]{2}{*}{ Factors } & \multirow{2}{*}{$\frac{\text { Univariate }}{p}$} & \multicolumn{3}{|c|}{ Multivariate } \\
\hline & & Hazard ratio & $95 \% \mathrm{Cl}$ & $p$ \\
\hline Preoperative mGPS-0 (vs. 1-2) & 0.002 & 0.291 & $0.092-0.927$ & 0.037 \\
\hline RO/1 resection (vs. R2) & 0.033 & 0.323 & $0.124-0.840$ & 0.021 \\
\hline Age < 62 (vs. $\geq 62)$ & 0.038 & 0.409 & $0.150-1.113$ & 0.080 \\
\hline CA19-9 decrease rate $\geq 89 \%$ (vs. $<89 \%$ ) & 0.047 & & & \\
\hline Locally advanced (vs. metastatic) & 0.982 & & & \\
\hline Tumour location Ph (vs. Pbt) & 0.249 & & & \\
\hline Pre-treatment mGPS-0 (vs. 1-2) & 0.910 & & & \\
\hline $\begin{array}{l}\text { Preoperative treatment duration } \\
>11 \text { months (vs. < } 11 \text { months) }\end{array}$ & 0.689 & & & \\
\hline RECIST PR (vs. SD) & 0.817 & & & \\
\hline ASA-PS 1-2 (vs. 3) & 0.321 & & & \\
\hline Morbidity $C-D$ grade $\leq \|$ & 0.415 & & & \\
\hline pT1-2 (vs. pT3-4) & 0.404 & & & \\
\hline pNO (vs. pN1) & 0.217 & & & \\
\hline pTNM stage I-II (vs. III-IV) & 0.240 & & & \\
\hline Histology well (vs. moderate-poor) & 0.156 & & & \\
\hline Evans grade III-IV (vs. I-IIb) & 0.418 & & & \\
\hline
\end{tabular}


15.9 months, which was significantly worse than the 31.0 months for those with mGPS-0. A prognosis with malignant tumours in general is markedly affected by the nutritional status of the individual and the extent of the tumour-associated inflammation. A number of studies have examined the relationship between inflammation-based scores and the prognosis of cancer patients [28-32]. In pancreatic cancer, mGPS correlates strongly with prognosis in patients with resectable and unresectable PC [28, 30]. However, in this study, pre-treatment scores including mGPS did not have a significant association with OS. The reason for this was unclear, but preoperative MGPS, which reflects both the residual tumour-related and host-related inflammatory state after non-surgical treatment, may be more useful to guide prognosis than pre-treatment mGPS in patients who are candidates for CS.

In a multicentre survey conducted in Japan, more than 240 days of preoperative non-surgical treatment was the only independent factor associated with favourable survival in patients receiving CS, by multivariate analysis [6]. Although we did not find a significant relationship between the duration of preoperative treatment and OS, a longer duration of preoperative treatment may be associated with better patient selection allowing for the identification of progressive disease or poor surgical candidates, and consequently may lead to a better prognosis of patients [6]. However, duration of preoperative treatment is part of the OS from treatment initiation, and in a sense is an outcome rather than a prognostic factor. In addition, patients enrolled in the abovementioned study received gemcitabine-based or S1 chemotherapy between 2001 and 2009 before approval of the use of gemcitabine plus nab-paclitaxel and FOLFIRINOX in Japan. The optimal timing of CS following intensive chemotherapy remains an essential clinical question that needs to be investigated further.

Adding ITCZ to cytotoxic chemotherapy was based on previous reports that indicated that ITCZ enhances the effect of taxanes by inhibiting P-glycoprotein, which has a critical role in chemoresistance $[14,16]$. ITCZ is also a potent antagonist of the Hedgehog pathway, which might promote tumorigenesis by signalling in a paracrine manner from the tumour to the surrounding stroma or in cancer stem cells [13]. Inappropriate activation of the Hedgehog signalling pathway has been implicated in the development of several types of cancer, including lung, prostate, breast, pancreas, brain, and skin [33, 34]. Furthermore, ITCZ inhibits endothelial cell cycle progression at the G1 phase in vitro and blocks vascular endothelial growth factor/basic fibroblast growth factor-dependent angiogenesis in vivo [15]. Thirty-eight metastatic PC patients who had a history of progression during or after prior chemotherapy had an additional survival of 11.4 months with ITCZ containing chemotherapy in a second line or more setting [16] (four of those patients underwent CS and were included in this study).

In an analysis of 12 patients undergoing CS, Kato et al. reported in 2011 that there were no five-year survivors in the four patients with metastatic disease, and eight patients with locally advanced disease had a significantly better prognosis than those with distant metastasis [4]. In 2018, Asano et al. from the same institute compared the survival between a locally advanced group $(n=26)$ and a distant metastasis group $(n=8)$, and there was a significant difference between the two groups in the generalised Wilcoxon test, but no significant difference was observed in the log-rank test [10]. They suggested that a statistical difference by the log-rank test may become significant if a larger number of patients with metastatic disease were included. Contrary to their results, OS was similar regardless of the presence of distant metastases in the present study. Moreover, all four patients who achieved five-year survival had an initially metastatic disease (two patients each with liver metastases and peritoneal seeding). Our study included more patients with metastatic PC than previous studies on CS and suggests that intensive modified chemotherapy using ITCZ may be effective in eradicating systemic disease.

There were several limitations of this study. First, it was a single-centre study with a small single-arm cohort. Further case accumulation and validation with a large number of cases will be needed to confirm our results. Moreover, to further investigate the role of CS, including the difference between each unresectable factor, it would be better to enrol a control group who responded well to non-surgical treatment but did not have CS. Second, mGPS may change with the timing of the blood test, especially in patients near the threshold boundary, which limits reliable patient stratification. Third, the indication for CS was not strictly controlled because this study was retrospective. Finally, the preoperative treatment regimen varied among patients, and therefore the overall conversion rate was unclear.

\section{Conclusions}

The conversion surgery is often a technically challenging and invasive therapy with significant morbidity. In addition, it may not necessarily provide a survival benefit in the era of effective chemotherapy. Our results show that preoperative MGPS after non-surgical treatment can help to identify patients who may have an extended survival with CS-combined multidisciplinary therapy. Further studies are needed to investigate the most appropriate determinant in selecting patients for CS.

\section{The authors declare no conflict of interest.}

\section{References}

1. Bray F, Ferlay J, Soerjomataram I, Siegel RL, Torre LA, Jemal A. Global cancer statistics 2018: GLOBOCAN estimates of incidence and mortality worldwide for 36 cancers in 185 countries. CA Cancer J Clin 2018; 68: 394-424.

2. Rahib L, Smith BD, Aizenberg R, Rosenzweig AB, Fleshman JM, Matrisian LM. Projecting cancer incidence and deaths to 2030: the unexpected burden of thyroid, liver, and pancreas cancers in the United States. Cancer Res 2014; 74: 2913-2921.

3. Vincent A, Herman J, Schulick R, Hruban RH, Goggins M. Pancreatic cancer. Lancet 2011; 378: 607-620.

4. Kato K, Kondo S, Hirano S, Tanaka E, Shichinohe T, Tsuchikawa T, Matsumoto J. Adjuvant surgical therapy for patients with initially- 
unresectable pancreatic cancer with long-term favorable responses to chemotherapy. J Hepatobiliary Pancreat Sci 2011; 18: 712-716.

5. Strobel O, Berens V, Hinz U, et al. Resection after neoadjuvant therapy for locally advanced, "unresectable" pancreatic cancer. Surgery 2012; 152 (3 Suppl 1): S33-42.

6. Satoi S, Yamaue H, Kato K, et al. Role of adjuvant surgery for patients with initially unresectable pancreatic cancer with a longterm favorable response to non-surgical anti-cancer treatments: results of a project study for pancreatic surgery by the Japanese Society of Hepato-Biliary-Pancreatic Surgery. J Hepatobiliary Pancreat Sci 2013; 20: 590-600.

7. Opendro SS, Satoi S, Yanagimoto H, et al. Role of adjuvant surgery in initially unresectable pancreatic cancer after long-term chemotherapy or chemoradiation therapy: survival benefit? J Hepatobiliary Pancreat Sci 2014; 21: 695-702.

8. Amano R, Kimura K, Nakata B, Yamazoe S, Motomura H, Yamamoto A, Tanaka S, Hirakawa K. Pancreatectomy with major arterial resection after neoadjuvant chemoradiotherapy gemcitabine and S-1 and concurrent radiotherapy for locally advanced unresectable pancreatic cancer. Surgery 2015; 158: 191-200.

9. Hackert T, Sachsenmaier M, Hinz U, et al. Locally advanced pancreatic cancer: neoadjuvant therapy with folfirinox results in re sectability in $60 \%$ of the patients. Ann Surg 2016; 264: 457-463.

10. Asano T, Hirano S, Nakamura T, et al. Survival benefit of conversion surgery for patients with initially unresectable pancreatic cancer who responded favorably to nonsurgical treatment. J Hepatobiliary Pancreat Sci 2018; 25: 342-350.

11. Von Hoff DD, Ervin T, Arena FP, et al. Increased survival in pancreatic cancer with nab-paclitaxel plus gemcitabine. N Engl J Med 2013; 369: 1691-1703.

12. Conroy T, Desseigne F, Ychou M, et al. FOLFIRINOX versus gemcitabine for metastatic pancreatic cancer. N Engl J Med 2011; 364: 1817-1825.

13. Kim J, Tang JY, Gong R, et al. Itraconazole, a commonly used an tifungal that inhibits Hedgehog pathway activity and cancer growth. Cancer Cell 2010; 17: 388-399.

14. Takara K, Tanigawara Y, Komada F, Nishiguchi K, Sakaeda T, Okumura K. Cellular pharmacokinetic aspects of reversal effect of itraconazole on P-glycoprotein-mediated resistance of anticancer drugs. Biol Pharm Bull 1999; 22: 1355-1359.

15. Chong CR, Xu J, Lu J, Bhat S, Sullivan DJ Jr, Liu JO. Inhibition of an giogenesis by the antifungal drug itraconazole. ACS Chem Biol 2007; 2: 263-270.

16. Tsubamoto H, Sonoda T, Ikuta S, Tani S, Inoue K, Yamanaka N. Combination chemotherapy with itraconazole for treating metastatic pancreatic cancer in the second-line or additional setting. Anticancer Res 2015; 35: 4191-4196.

17. Japan Pancreas Society. General rules for the study of pancreatic cancer. $7^{\text {th }}$ ed. Kanehara \& Co., Ltd., Tokyo 2016.

18. Eisenhauer EA, Therasse P, Bogaerts J, et al. New response evaluation criteria in solid tumours: revised RECIST guideline (version 1.1). Eur J Cancer 2009; 45: 228-247.

19. Toiyama Y, Miki C, Inoue Y, Tanaka K, Mohri Y, Kusunoki M. Evaluation of an inflammation-based prognostic score for the identification of patients requiring postoperative adjuvant chemotherapy for stage II colorectal cancer. Exp Ther Med 2011; 2: 95-101.

20. Dindo D, Demartines N, Clavien PA. Classification of surgical complications: a new proposal with evaluation in a cohort of $6336 \mathrm{pa}$ tients and results of a survey. Ann Surg 2004; 240: 205-213.

21. Evans DB, Rich TA, Byrd DR, et al. Preoperative chemoradiation and pancreaticoduodenectomy for adenocarcinoma of the pancreas. Arch Surg 1992; 127: 1335-1339.

22. Schemper M, Smith TL. A note on quantifying follow-up in studies of failure time. Control Clin Trials 1996; 17: 343-346.

23. van Erkel AR, Pattynama PM. Receiver operating characteristic (ROC) analysis: basic principles and applications in radiology. Eur J Radiol 1998; 27: 88-94.

24. Kim E, Kim K, Kyu Chie E, Oh DY, Tae Kim Y. Chemoradiotherapy after gemcitabine plus erlotinib in patients with locally advanced pancreatic cancer. J BUON 2017; 22: 1046-1052.

25. Montes AF, Villarroel PG, Ayerbes MV, Gómez JC, Aldana GQ, Tuñas LV, Fernández MS, Fernández MJ. Prognostic and predictive mark- ers of response to treatment in patients with locally advanced unresectable and metastatic pancreatic adenocarcinoma treated with gemcitabine/nab-paclitaxel: results of a retrospective analysis. J Cancer Res Ther 2017; 13: 240-245.

26. Yoshida K, Iwashita T, Uemura S, et al. A multicenter prospective phase II study of first-line modified FOLFIRINOX for unresectable advanced pancreatic cancer. Oncotarget 2017; 8: 111346-111355.

27. Chan KK, Guo H, Beca JM, et al. Outcomes of FOLFIRINOX (FFX) and gemcitabine+nab-paclitaxel (GnP) in initially unresectable locally advanced pancreatic cancer (ULAPC): a population-based study. J Clin Oncol 2017; 35: abstr 394.

28. La Torre M, Nigri G, Cavallini M, Mercantini P, Ziparo V, Ramacciato $G$. The Glasgow prognostic score as a predictor of survival in patients with potentially resectable pancreatic adenocarcinoma. Ann Surg Oncol 2012; 19: 2917-2923.

29. Petrelli F, Barni S, Coinu A, Bertocchi P, Borgonovo K, Cabiddu M, Ghilardi M, Zaniboni A. The modified Glasgow prognostic score and survival in colorectal cancer: a pooled analysis of the literature. Rev Recent Clin Trials 2015; 10: 135-141.

30. Asama H, Suzuki R, Takagi T, et al. Evaluation of inflammation-based markers for predicting the prognosis of unresectable pancreatic ductal adenocarcinoma treated with chemotherapy. Mol Clin Oncol 2018; 9: 408-414.

31. Szor DJ, Roncon Dias A, Pereira MA, Ramos MFKP, Zilberstein B, Cecconello I, Ribeiro U Jr. Neutrophil-lymphocyte ratio is associated with prognosis in patients who underwent potentially curative resection for gastric cancer. J Surg Oncol 2018; 117: 851-857.

32. Zhao OT, Zhang XP, Zhang H, Duan GC. Prognostic role of platelet to lymphocyte ratio in esophageal cancer: a meta-analysis. Oncotarget 2017; 8: 112085-112093.

33. Thayer SP, di Magliano MP, Heiser PW, et al. Hedgehog is an early and late mediator of pancreatic cancer tumorigenesis. Nature 2003; 425: 851-856.

34. Gupta S, Takebe N, Lorusso P. Targeting the Hedgehog pathway in cancer. Ther Adv Med Oncol 2010; 2: 237-250.

\section{Address for correspondence}

\section{Shinichi Ikuta}

Department of Surgery

Meiwa Hospital

4-31 Agenaruo, Nishinomiya

Hyogo 663-8186, Japan

tel.: +81-798-47-1767

e-mail: g2s1002@gmail.com

Submitted: 29.11.2018

Accepted: $\quad 16.12 .2018$ 